\title{
Micromorfologia do solo e sua relação com atributos físicos e hídricos
}

\author{
Zigomar Menezes de Souza( ${ }^{(1)}$, José Marques Júnior ${ }^{(1)}$, Miguel Cooper ${ }^{(2)}$ e Gener Tadeu Pereira ${ }^{(3)}$
}

\begin{abstract}
(1)Universidade do Estado de São Paulo (Unesp), Fac. de Ciências Agrárias e Veterinárias, Dep. de Solos e Adubos, Via de acesso Prof. Paulo Donato Castellane, s/no, CEP 14884-900 Jaboticabal, SP. E-mail: zigomar@fcav.unesp.br, marques@fcav.unesp.br (2)Universidade de São Paulo, Escola Superior de Agricultura Luiz de Queiroz, Dep. de Solos e Nutrição de Plantas, Av. Pádua Dias, no 11, CEP 13418-900 Piracicaba, SP. E-mail: mcooper@carpa.ciagri.usp.br (3)Unesp, Fac. de Ciências Agrárias e Veterinárias, Dep. de Ciências Exatas. E-mail: genertp@fcav.unesp.br
\end{abstract}

Resumo - O objetivo deste trabalho foi avaliar a relação entre atributos micromorfológicos e físicos de um Latossolo Vermelho eutroférrico, sob cultivo de cana-de-açúcar. As amostras de solo foram coletadas nos pontos de cruzamento de uma malha, com intervalos regulares de $10 \mathrm{~m}$, perfazendo um total de 100 pontos, nos horizontes A1 e AB. Os altos valores da densidade do solo, microporosidade e resistência do solo à penetração, e os baixos valores da macroporosidade, porosidade total e condutividade hidráulica do solo saturado, principalmente no horizonte AB, indicaram compactação confirmada pela análise de imagens (micromorfologia).

Termos para indexação: Saccharum officinarum, resistência do solo à penetração, condutividade hidráulica do solo saturado, análise de imagens.

\section{Soil micromorphology and its relation with physical and hydrical attributes}

\begin{abstract}
The objective of this work was to evaluate the relationship between soil micromorphological and physical attributes in an Oxisol (Rhodic Eutrudox) under sugarcane. Soil samples were collected at the intersection points of a regular grid, with $10 \mathrm{~m}$ intervals, comprising a total of 100 points, in the horizons A1 and AB. The higher values for bulk density, microporosity and soil penetration resistance and lower values for macroporosity, total porosity and saturated hydraulic conductivity mainly in the AB horizon, indicated compaction in this horizon, confirmed by image analysis (micromorphology).
\end{abstract}

Index terms: Saccharum officinarum, soil penetration resistance, saturated hydraulic conductivity, image analysis.

\section{Introdução}

O grau de compactação do solo é importante na avaliação do manejo da cultura da cana-de-açúcar, por ser um sistema altamente mecanizado. A utilização intensiva de máquinas e implementos agrícolas no nordeste do Estado de São Paulo, no cultivo da cana-de-açúcar, tem contribuído para aumentar as áreas com problemas de compactação, provavelmente pela ausência de cronograma de trabalho definido ou de modelos capazes de estimar a capacidade de suporte do solo (Casagrande, 2002).

O uso intensivo do Latossolo Vermelho eutroférrico, no nordeste do Estado de São Paulo, com o cultivo da cana-de-açúcar, preparo superficial excessivo e queima dos resíduos, modifica significativamente os atributos físicos do solo (Souza et al., 2004). As principais alterações são diminuição do volume de macroporos, do tamanho de agregados, da taxa de infiltração de água e o aumento da resistência à penetração de raízes e da densidade do solo (Dias Junior \& Pierce, 1996; Figueiredo et al., 2000; Utset \& Cid, 2001). A descrição e a quantificação detalhada das feições, utilizando técnicas micromorfológicas e de análise de imagens, permitem obter evidências acerca dessas mudanças nos atributos físicos do solo (Castro et al., 2003).

O movimento de água no solo é mais dependente da estrutura do que da textura do solo, porque é afetado pelo sistema de poros existentes (Ahuja et al., 1984). Soares et al. (2005a), estudando as alterações físicas e morfológicas em solos cultivados com citros e cana-deaçúcar sob sistema tradicional de manejo, observaram por meio de análises micromorfológicas, redução da macroporosidade e infiltração de água no solo, sendo esta redução mais intensa nos solos cultivados com canade-açúcar. 
A micromorfologia é uma técnica muito útil no estudo da gênese do solo e na avaliação e no monitoramento de diversas práticas agrícolas. Com o auxílio das técnicas de processamento e análise digital de imagem, a micromorfologia é capaz de fornecer resultados de porosidade e permeabilidade com precisão, além de possibilitar a visualização das alterações estruturais causadas pela compactação e adensamento (Castro et al., 2003).

O uso de técnicas de análise de imagens nos estudos quantitativos e qualitativos de estrutura do solo tem se tornado mais comum, com maior acesso a equipamentos e programas, e maior número de pesquisadores na área. Essas técnicas, associadas à micromorfologia, permitem o estudo quantitativo de modificações estruturais em amostras indeformadas, depois de seu preparo e sua montagem em lâminas (Viana et al., 2004).

Considerando que mudanças nos atributos físicohídricos são, em grande parte, conseqüência de alterações na estrutura do solo (Drees et al., 1994), o objetivo deste trabalho foi avaliar a relação entre atributos micromorfológicos e físicos de um Latossolo Vermelho eutroférrico, sob cultivo de cana-de-açúcar.

\section{Material e Métodos}

A área de estudo localiza-se no nordeste do Estado de São Paulo, no Município de Guariba, SP (21ํํ'S, 48¹3'W; a $640 \mathrm{~m}$ de altitude). O clima da região, segundo a classificação de Köppen, é do tipo mesotérmico com inverno seco (Cwa), com precipitação média de $1.400 \mathrm{~mm}$, com chuvas concentradas no período de novembro a fevereiro.

O relevo é predominantemente suave ondulado com declividades médias variando de 3 a 8\%. O solo da área em estudo foi classificado como Latossolo Vermelho eutroférrico, textura muito argilosa (LVef) (Embrapa, 1999). A área apresenta histórico de cultivo intensivo de cana-de-açúcar por mais de 30 anos consecutivos.

O solo foi amostrado em 100 pontos de cruzamento de uma malha com intervalos regulares de $10 \mathrm{~m}$, em uma área de 100x100 m, nos horizontes A1 e AB. Foram abertas 100 trincheiras de $0,60 \mathrm{~m}$ de profundidade (0,3x0,3 m de largura), para a coleta de amostras nas quais foram determinados os atributos físicos. As amostras micromorfológicas foram coletadas em uma trincheira aberta no centro da área. As amostras indeformadas foram coletadas com auxílio de anéis volumétricos de 0,04 $\mathrm{m}$ de altura e 0,06 m de diâmetro, para determinação da microporosidade e da macroporosidade, utilizando a mesa de tensão. A porosidade total foi determinada segundo Embrapa (1997), a microporosidade, por secamento (-0,006 MPa) e a macroporosidade, por diferença entre a porosidade total e a microporosidade. A densidade do solo foi determinada conforme Embrapa (1997).

A resistência do solo à penetração foi determinada com o penetrômetro de impacto (modelo IAA/ Planalsucar-Stolf), com ângulo de cone de $30^{\circ}$ e os resultados transformados conforme Stolf (1991). Na avaliação da condutividade hidráulica do solo saturado no campo, empregou-se o permeâmetro de Guelph, modificado por Vieira (1998). Mediu-se a taxa constante de infiltração e calculou-se a condutividade hidráulica do solo saturado no campo (Kfs), de acordo com Reynolds \& Elrick (1985). Feitas as medições, os dados foram analisados segundo modelos matemáticos propostos por Vieira (1998).

Nas análises micromorfológicas, lâminas delgadas $(5 \times 7 \mathrm{~cm})$ foram feitas a partir de blocos impregnados com resina poliéster não saturada, diluída com monômero de estireno e misturada com pigmento fluorescente (Uvitex OB), que permite a distinção dos poros, quando iluminados com luz ultravioleta (Murphy, 1986). Imagens digitalizadas foram adquiridas a partir das lâminas delgadas e blocos polidos utilizando uma câmera CCD com resolução de $1.024 \times 768$ pixels e área por pixel de $156,25 \mu \mathrm{m}^{2}$.

As imagens foram processadas utilizando o programa Visilog 5.4 (Noesis). A porosidade total (PT) foi calculada como a soma das áreas de todos os poros dividida pela área total da imagem, em porcentagem. Os poros foram divididos em três grupos de acordo com sua forma: arredondados, alongados e complexos. Dois índices foram utilizados para determinar a forma dos poros:

$I_{1}=\frac{P^{2}}{(4 \pi A)}$

em que P é o perímetro do poro e A sua área, e

$I_{2}=\frac{\frac{1}{m} \sum_{i}\left(N_{I}\right)_{i}}{\frac{1}{n} \sum_{j}\left(D_{F}\right)_{j}}$ 
em que $\mathrm{N}_{\mathrm{I}}$ é o número de interceptos de um objeto na direção i $\left(i=0,45,90\right.$ e $\left.135^{\circ}\right)$, $D_{F}$ é o diâmetro de Feret de um objeto na direção j $\left(\mathrm{j}=0\right.$ e $\left.90^{\circ}\right)$, m é o número de i direções e n é o número de j direções. O índice $\mathrm{I}_{2}$ é utilizado como complemento ao índice $\mathrm{I}_{1}$ para obter precisão mais alta na separação entre os grupos de formas arredondados, alongados e complexos.

A partir de jogo de imagens nas quais as principais formas dos poros podem ser observadas, procede-se à identificação dos poros. Canais, cavidades isoladas e poros tubulares pertencem à categoria de poros com índice de forma $\mathrm{I}_{1} \leq 5$ e definem a classe morfológica Arr de poros arredondados. Os poros que resultam da assembléia de agregados ou de partículas elementares e de poros de diversos tipos, interconectados, formando, nos dois casos, uma rede poral de tamanho grande de formas muito contornadas, pertenciam à categoria de poros caracterizada por um índice de forma $\mathrm{I}_{1}>25$ e define os poros de forma complexa (Comp). Dentro da gama de índices de forma $\mathrm{I}_{1}$, compreendido entre 5 e 25, coexistem poros alongados e poros de forma complexa, de tamanho menor, em geral, que os precedentes. Neste caso, o índice $I_{2}$ foi elaborado para separar os limites do índice $\mathrm{I}_{1}$, o qual permitiu melhor discriminar os poros alongados (Alon) e os poros complexos (Comp); os primeiros têm um $\mathrm{I}_{2} \leq 2,2$ e os segundos um $\mathrm{I}_{2}>2,2$.

As três classes de forma, arredondados (Arr), alongados (Alon) e complexos (Comp), foram cruzadas com as classes de tamanho dos poros na escala utilizada. Desta forma, foram definidos nove tipos de poros, segundo sua forma e tamanho: poros arredondados pequenos, médios e grandes ( $\operatorname{Arr}_{\mathrm{p}}, \operatorname{Arr}_{\mathrm{m}}, \mathrm{Arr}_{\mathrm{g}}$ ); alongados pequenos, médios e grandes $\left(\mathrm{Alon}_{\mathrm{p}}\right.$, $\left.\mathrm{Alon}_{\mathrm{m}}, \mathrm{Alon}_{\mathrm{g}}\right)$ e complexos pequenos, médios e grandes (Comp, $\mathrm{Comp}_{\mathrm{m}}$, Compg). As classes de tamanho foram definidas da seguinte maneira: poros pequenos, aqueles que ocupam áreas entre $1,56 \times 10^{-4}$ e $1,56 \times 10^{-2} \mathrm{~mm}^{2}$; poros médios, áreas entre $1,56 \times 10^{-2}$ e $0,156 \mathrm{~mm}^{2}$ e poros grandes, áreas maiores do que $0,156 \mathrm{~mm}^{2}$.

A análise estatística constou dos dados de 100 coletas simples ao acaso, nos horizontes A1 e AB, com distância de $10 \mathrm{~m}$, que foram avaliados estatisticamente pelo confronto de médias, duas a duas, pelo teste t de Student a $5 \%$ de probabilidade, por meio do SAS Institute (1995).

\section{Resultados e Discussão}

Os valores médios de resistência do solo à penetração pertencem às classes alta no horizonte $\mathrm{A} 1$ e muito alta no horizonte AB, de acordo com USDA (1993). Houve aumento da resistência do solo à penetração do horizonte A1 para o horizonte AB (Tabela 1), com valores considerados restritivos ao desenvolvimento radicular, segundo Grant \& Lafond (1993), os quais afirmam que valores compreendidos entre 1,5 e 3,0 MPa são considerados críticos e dificultam o desenvolvimento radicular das culturas.

A condutividade hidráulica do solo saturado (Tabela 1) apresenta valores médios pertencentes à classe lenta a moderada para o horizonte A1 e lenta no horizonte AB (USDA, 1993). Os valores médios da densidade do solo foram de 1,38 a 1,41 $\mathrm{kg} \mathrm{dm}^{-3}$, considerados altos, segundo Arshad et al. (1996), que relatam que valores acima de $1,40 \mathrm{~kg} \mathrm{dm}^{-3}$ restringem o crescimento radicular em solo argiloso. Os valores altos da densidade do solo e resistência à penetração e os valores baixos de permeabilidade são conseqüências do tráfego intensivo de máquinas na cultura da cana-de-açúcar. Segundo Salire et al. (1994) e Hakansson \& Voorhees (1998), sistemas com pouco revolvimento do solo e tráfego de máquinas pesadas podem promover compactação do solo até $0,4 \mathrm{~m}$.

A análise da porosidade média total na trincheira, a partir dos blocos polidos e lâminas delgadas, indicou aumento gradativo da porosidade total entre os horizontes $\mathrm{AB}$ e $\mathrm{Bw} 3$, passando de uma porosidade média de $13 \%$ no horizonte $\mathrm{AB}$, para $34 \%$, no $\mathrm{Bw} 3$ (Figura 1). O horizonte AB apresentou o menor valor para porosidade total (13\%), indicando maior compactação nesse horizonte. Nos locais onde ocorre ação do tráfego intensivo de máquinas agrícolas (horizontes A1 e AB), os valores de porosidade total foram menores em relação aos horizontes que não sofreram ação do manejo (horizontes Bw2 e Bw3), corrobo-

Tabela 1. Valores médios de atributos físicos em Latossolo Vermelho eutroférrico.

\begin{tabular}{lrr}
\hline Atributo físico $^{(1)}$ & Horizonte & Horizonte \\
& $\mathrm{A} 1$ & $\mathrm{AB}$ \\
\hline Condutividade hidráulica $\left(\mathrm{mm} \mathrm{h}^{-1}\right)$ & $30,11 \mathrm{a}$ & $23,48 \mathrm{~b}$ \\
Densidade do solo $\left(\mathrm{kg} \mathrm{dm}^{-3}\right)$ & $1,38 \mathrm{a}$ & $1,41 \mathrm{a}$ \\
Macroporosidade $\left(\mathrm{m}^{3} \mathrm{~m}^{-3}\right)$ & $0,15 \mathrm{a}$ & $0,13 \mathrm{a}$ \\
Microporosidade $\left(\mathrm{m}^{3} \mathrm{~m}^{-3}\right)$ & $0,36 \mathrm{~b}$ & $0,40 \mathrm{a}$ \\
Porosidade total $\left(\mathrm{m}^{3} \mathrm{~m}^{-3}\right)$ & $0,50 \mathrm{a}$ & $0,46 \mathrm{~b}$ \\
Resistência do solo à penetração $(\mathrm{MPa})$ & $4,40 \mathrm{~b}$ & $5,11 \mathrm{a}$ \\
\hline
\end{tabular}

${ }^{(1)}$ Médias de 100 observações seguidas da mesma letra, na linha, não diferem entre si pelo teste t a $5 \%$ de probabilidade. 
rando os resultados de Pagliai et al. (1983) e Cooper (1999).

Os altos valores médios da densidade do solo, microporosidade e resistência do solo à penetração e os baixos valores de macroporosidade e condutividade hidráulica do solo saturado foram verificados no horizonte AB (Tabela 1), concordando com os resultados alcançados por meio da análise de imagem, em que se observou menor porosidade total e maior distribuição de poros arredondados neste horizonte (Figura 1). A mobilização do solo e o tráfego de máquinas e implementos agrícolas, comuns no cultivo da cana-deaçúcar, modificaram o tamanho de agregados, com aumento na proporção de microporos em relação aos macroporos, corroborando resultados obtidos por Bullock \& Thomasson (1979) e Silva \& Mielniczuk (1998).

Todas as formas de poros estão representadas em todos os horizontes, e o horizonte $\mathrm{AB}$ foi o que apresentou menor porosidade total (13\%) e maior quantidade de poros arredondados responsáveis por menor condução de água ao longo do perfil do solo, em razão de provável baixa conectividade entre esses poros (Figura 1). O horizonte Bw3 apresentou maior porosidade total (34\%), assim como maior número de poros complexos, conduzindo maior quantidade de água ao longo do perfil. Maior quantidade de poros arredondados no tratamento com cultivo em relação ao tratamento não cultivado foi observado por Pagliai et al. (1983).

No horizonte $\mathrm{AB}$, ocorreu a menor condutividade hidráulica do solo saturado (Tabela 1), estando esse resultado de acordo com a menor porosidade total e distribuição de poros arredondados (Figuras 1 e 2). Já nos horizontes Bw2 e Bw3, observaram-se maiores valores de condutividade hidráulica do solo saturado (Tabela 1). Isto se deve, provavelmente, à maior quantidade de porosidade total e às formas complexas de seus poros, que promovem maior movimentação da água ao longo do perfil do solo (Figuras 1 e 2). Maior movimento de água ao longo do perfil por meio dos poros alongados também foram observados por Fox et al. (2004). O mesmo comportamento foi constatado por Cooper (1999) nos horizontes Bw, em virtude do maior desenvolvimento da porosidade complexa nesses horizontes.

As observações microscópicas das lâminas delgadas revelam diferenciações da geometria do espaço poroso, interferindo na condutividade hidráulica do solo saturado, em conseqüência de alterações causadas pelo manejo, concordando com os resultados de Soares et al. (2005b).
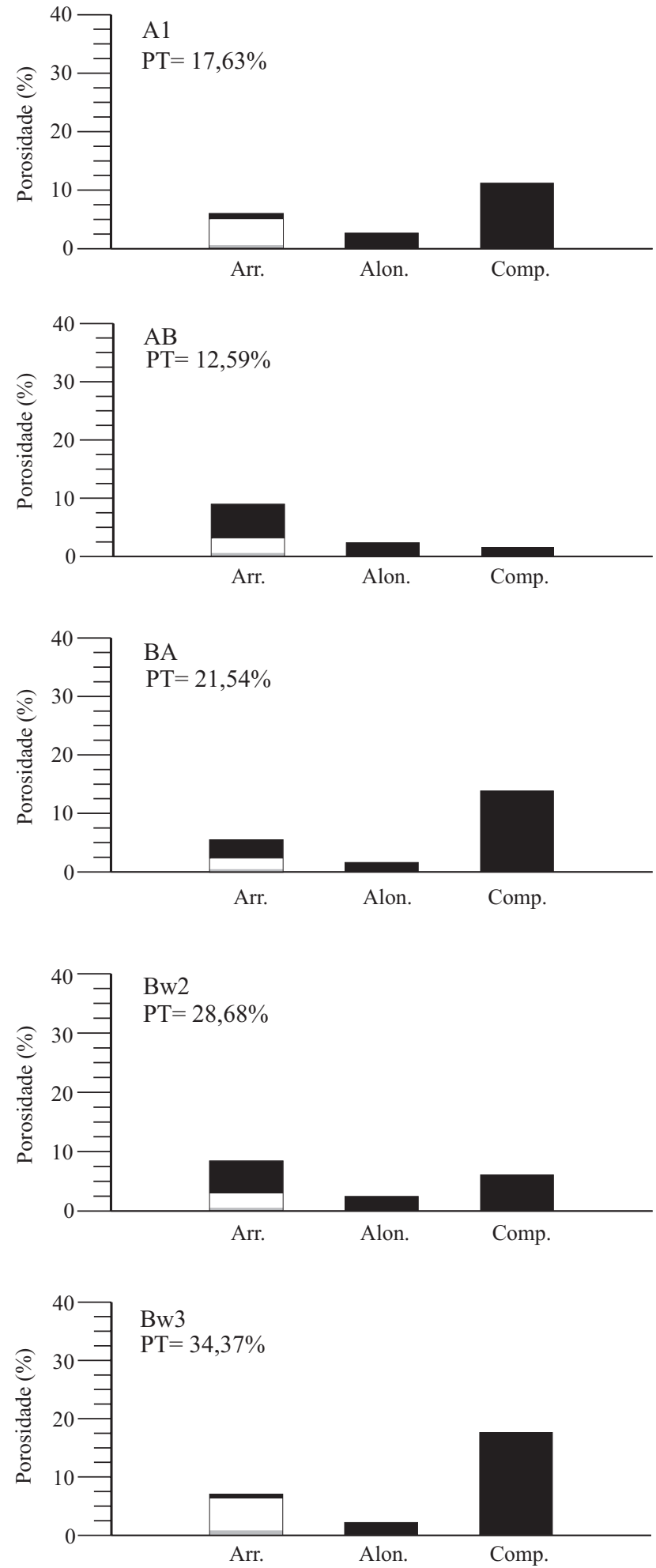

Figura 1. Distribuição da porosidade total (PT), segundo a forma (Arr: arredondados; Alon: alongados; Comp: complexos) e o tama-

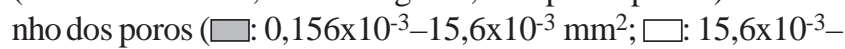
156x10-3 $\left.\mathrm{mm}^{2}, \mathbf{\square}:>156 \times 10^{-3} \mathrm{~mm}^{2}\right)$, nos horizontes A1 (0-26 cm), AB(26-62 cm), BA(62-98 cm), Bw2(98-159 cm)eBw3 $\left(159^{+} \mathrm{cm}\right)$. 


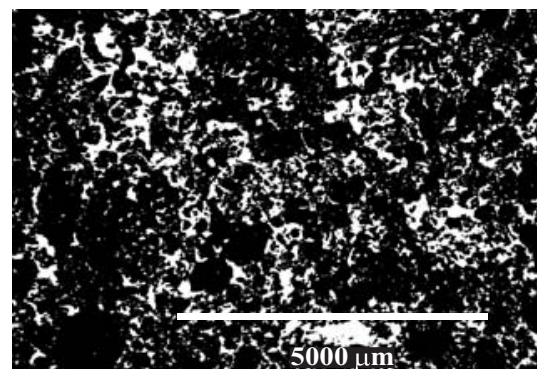

A 1

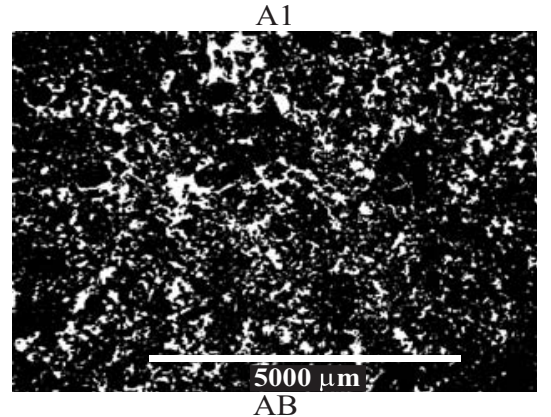

$\mathrm{AB}$

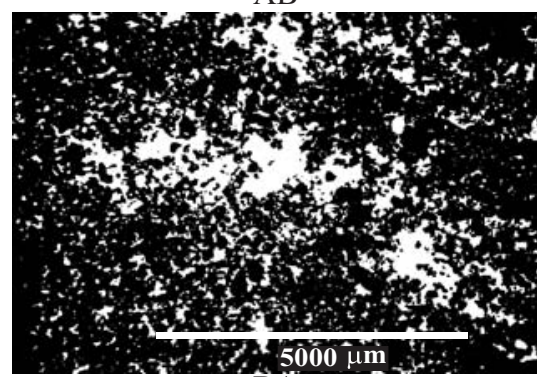

BA

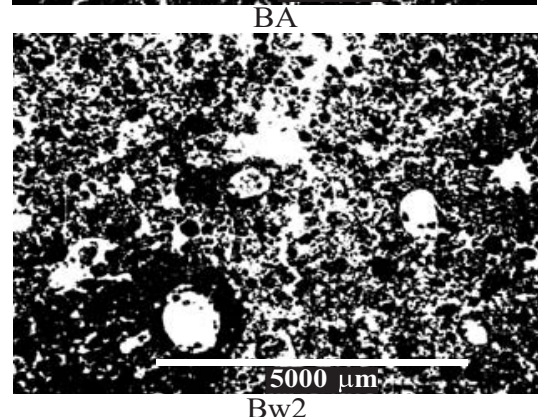

Bw2

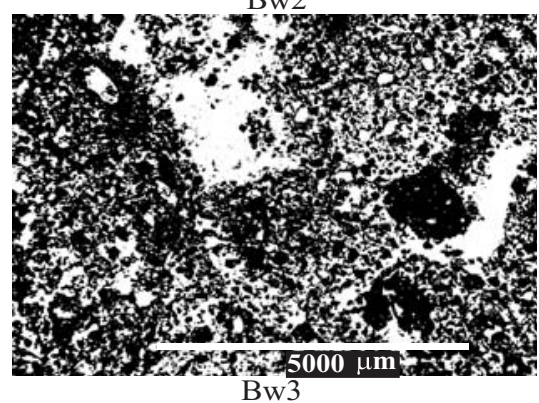

Bw3

Figura 2. Imagens binárias de um Latossolo Vermelho eutroférrico (os poros aparecem em branco e a fase sólida em preto), correspondentes aos horizontes A1 (0-26 cm), AB (26$62 \mathrm{~cm})$, BA (62-98 cm), Bw2 (98-159 cm) e $\mathrm{Bw} 3\left(159^{+} \mathrm{cm}\right)$.

\section{Conclusões}

1. Os atributos físico-hídricos mostram compactação nos horizontes $\mathrm{A} 1 \mathrm{e} \mathrm{AB}$.

2. A análise de imagem confirma a compactação do solo nesses horizontes.

\section{Referências}

AHUJA, L.R.; NANEY, J.W.; GREEN, R.E.; NIELSEN, D.R. Macroporosity to characterize spatial variability of hydraulic conductivity and effects of land management. Soil Science Society of America Journal, v.48, p.699-702, 1984.

ARSHAD, M.A.; LOWERY, B.; GROSSMAN, B. Physical tests for monitoring soil quality. In: DORAN, J.W.; JONES, A.J. (Ed.). Methods for assessing soil quality. Madison: Soil Science Society of America, 1996. p.123-142. (SSSA. Special publication, 49).

BULLOCK, P.; THOMASSON, A.J. Rothamsted studies of soil structure. II. Measurement and characterization of macroporosity by image analysis and comparison with data from water retention measurements. Journal of Soil Science, v.30, p.391-414, 1979.

CASAGRANDE, A.A. Compactação e manejo do solo na cultura da cana-de-açúcar. In: MORAES, M.H.; MÜLLER, M.M.L.; FOLONI, J.S.S. (Ed.). Qualidade física do solo: métodos de estudo-sistemas de preparo e manejo do solo. Jaboticabal: Funep, 2002. p.150-197.

CASTRO, S.S. de; COOPER, M.; SANTOS, M.C.; VIDALTORRADO, P. Micromorfologia do solo: bases e aplicações. In: CURI, N.; MARQUES, J.J.; GUILHERME, L.R.G.; LIMA, J.M. de; LOPES, A.S.; ALVAREZ VENEGAS, V.H. (Ed.). Tópicos em ciência do solo. Viçosa: Sociedade Brasileira de Ciência do Solo, 2003. v.3, p.107-164.

COOPER, M. Influência das condições físico-hídricas nas transformações estruturais entre horizonte B latossólico e B textural sobre diabásio. 1999. 132p. Tese (Doutorado) Universidade de São Paulo, Piracicaba.

DIAS JUNIOR, M. de S.; PIERCE, F.J. Revisão de literatura: o processo de compactação do solo e sua modelagem. Revista Brasileira de Ciência do Solo, v.20, p.175-182, 1996.

DREES, L.R.; KARATHANASIS, A.D.; WILDING, L.P.; BLEVINS, R.L. Micromorphological characteristics of long-term no-till and conventionally tilled soils. Soil Science Society of America Journal, v.58, p.508-517, 1994.

EMBRAPA. Centro Nacional de Pesquisa de Solos (Rio de Janeiro, RJ). Manual de métodos de análise de solo. 2.ed. Rio de Janeiro, 1997. 212p.

EMBRAPA. Centro Nacional de Pesquisa de Solos (Rio de Janeiro, RJ). Sistema brasileiro de classificação de solos. Brasília: Embrapa-SPI; Rio de Janeiro: Embrapa-CNPS, 1999. 412p.

FIGUEIREDO, L.H.A.; DIAS JUNIOR, M. de S.; FERREIRA, M.M. Umidade crítica de compactação e densidade do solo máxima em resposta a sistemas de manejo num Latossolo Roxo. Revista Brasileira de Ciência do Solo, v.24, p.487-493, 2000.

FOX, D.M.; BRYAN, R.B.; FOX, C.A. Changes in pore characteristics with depth for structural crusts. Geoderma, v.120, p.109-120, 2004. 
GRANT, C.A.; LAFOND, G.P. The effects of tillage systems and crop sequences on soil bulk density and penetration resistance on a clay soil in Southern Saskatchewan. Canadian Journal of Soil Science, v.73, p.223-232, 1993.

HAKANSSON, I.; VOORHEES, W.B. Soil compaction. In: LAL, R.; BLUM, W.H.; VALENTIN, C.; STEWART, B.A. (Ed.). Methods for assessment of soil degradation. Boca Raton: CRC Press, 1998. p.167-179.

MURPHY, C.P. Thin section preparation of soils and sediments. Berkhamsted, Herts, UK: AB Academic Pub., 1986. 160p.

PAGLIAI, M.; LA MARCA, M.; LUCAMANTE, G. Micromorphometric and micromorphological investigations of a clay loam soil in viticulture under zero and conventional tillage. Journal of Soil Science, v.34, p.391-403, 1983.

REYNOLDS, W.D.; ELRICK, D.E. In situ measurement of fieldsaturated hydraulic conductivity, sorptivity, and the alpha-parameter using the Guelph permeameter. Soil Science, v.140, p.292-302, 1985.

SALIRE, E.V.; HAMMEL, J.E.; HARDCASTLE, J.H. Compression of intact subsoils under short-duration loading. Soil and Tillage Research, v.31, p.235-248, 1994.

SAS INSTITUTE (Cary, Estados Unidos). Statistical analysis system for Windows: computer program manual. Cary, 1995. 705p.

SILVA, I.F.; MIELNICZUK, J. Sistemas de cultivo e características do solo afetando a estabilidade de agregados. Revista Brasileira de Ciência do Solo, v.22, p.311-317, 1998.
SOARES, J.L.N.; ESPINDOLA, C.R.; FOLONI, L.L. Alteração física e morfológica em solos cultivados com citros e cana-de-açúcar, sob sistema tradicional de manejo. Ciência Rural, v.35, p.353-359, 2005a.

SOARES, J.L.N.; ESPINDOLA, C.R.; PEREIRA, W.L.M. Physical properties of soils under intensive agricultural management. Scientia Agricola, v.62, p.165-172, 2005b.

SOUZA, Z.M. de; MARQUES JÚNIOR, J.; PEREIRA, G.T. Variabilidade espacial da estabilidade de agregados e matéria orgânica em solos de relevos diferentes. Pesquisa Agropecuária Brasileira, v.39, p.491-499, 2004.

STOLF, R. Teoria e teste experimental de fórmulas de transformação dos dados de penetrômetro de impacto em resistência do solo. Revista Brasileira de Ciência do Solo, v.15, p.229-235, 1991.

USDA. Soil Survey Division Staff. Soil survey manual. Washington, 1993. 437p. (Agriculture handbook, 18).

UTSET, A.; CID, G. Soil penetrometer resistance spatial variability in a ferralsol at several soil moisture conditions. Soil and Tillage Research, v.61, p.193-202, 2001.

VIANA, J.H.M.; FERNANDES FILHO, E.I.; SCHAEFER, C.E.G.R. Efeitos de ciclos de umedecimento e secagem na reorganização da estrutura microgranular de latossolos. Revista Brasileira de Ciência do Solo, v.28, p.11-19, 2004.

VIEIRA, S.R. Permeâmetro: novo aliado na avaliação de manejo do solo. O Agronômico, v.50, p.32-33, 1998.

Recebido em 5 de novembro de 2004 e aprovado em 9 de agosto de 2005 